\title{
Growth Factor Binding on Heparin Mimetic Peptide Nanofibers
}

\author{
Rashad Mammadov, Busra Mammadov, Mustafa O. Guler,* and Ayse B. Tekinay* \\ Institute of Materials Science and Nanotechnology, National Nanotechnology Research Center (UNAM), Bilkent University, Ankara \\ 06800, Turkey
}

Supporting Information

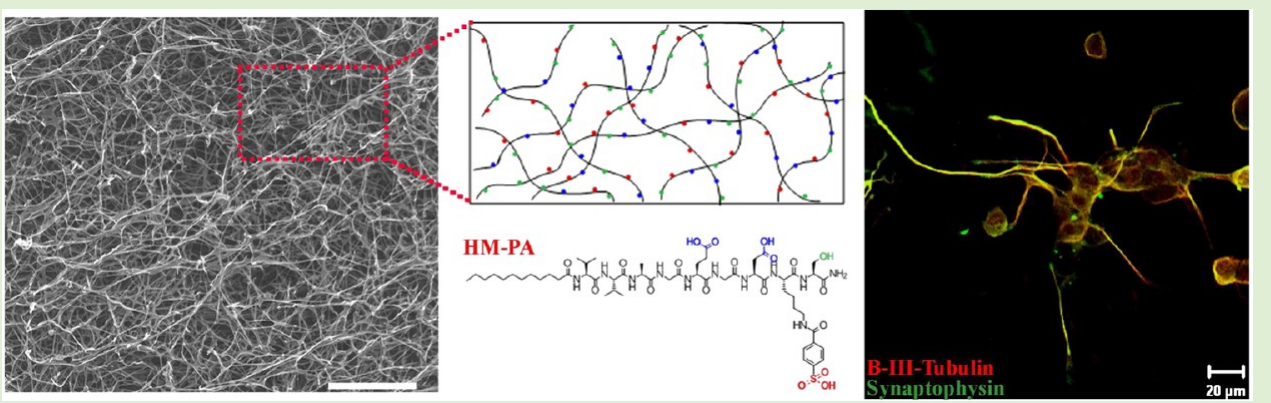

ABSTRACT: Immobilization of growth factors in scaffolds is important for controlling their dose and bioactivity for regenerative medicine applications. Although numerous covalent and noncovalent immobilization strategies have been proposed, better growth factor loading and dose control inside the scaffold is necessary. Nature of the binding site on the growth factor interacting with scaffold is critical for preserving and achieving maximal growth factor functionality, which has been a relatively less emphasized issue in previous studies. We recently reported heparin mimetic peptide nanofibers, which mimic chemistry of heparan sulfates. Heparin mimetic nanofibers were shown to bind to vascular endothelial growth factor (VEGF) and direct endothelial cells to angiogenesis. Here, we further investigated interactions between heparin mimetic peptide nanofibers and growth factors. We tested bioactivity of the nanofiber bound growth factors in order to understand the potential use of these peptide nanofiber scaffolds as analogues of heparan sulfates. We observed that heparin mimetic peptide nanofibers demonstrate better binding profiles to VEGF, hepatocyte growth factor (HGF), and fibroblast growth factor-2 (FGF-2) than control peptide nanofibers. We also identified that the heparin-binding domain of VEGF is critical for its interaction with these nanofibers. However, the heparin-binding site is not indispensable for binding of all growth factors to nanofibers. We also showed that binding of growth factors to nanofibers does not cause any loss in bioactivity through in vitro cell culture assays with PC-12 cells. These results reveal that heparin mimetic peptide nanofibers can effectively mimic heparan sulfates in extracellular matrix and provide an optimal milieu for spatial presentation of important growth factors. These properties make peptide nanofiber scaffolds promising materials for regenerative medicine applications through efficient and precisely controlled growth factor delivery.

\section{INTRODUCTION}

Functional biomaterials can aid efforts to control cell behavior and promote tissue regeneration. New-generation biomaterials differ from conventional ones in terms of controlling dose and bioactivity of delivered molecules (e.g., growth factors) more precisely rather than acting as mere scaffolds. ${ }^{1}$ Immobilization of growth factors either covalently or noncovalently to a scaffold provides spatial distribution of growth factors inside the scaffold. Although covalent immobilization enables prolonged release of growth factors, specificity of coupling site on the growth factors is difficult to achieve, and proteins may lose their bioactivity during coupling process. ${ }^{2}$ Materials can also be programmed to interact with growth factors through decoration of specific binding sites that interact with growth factors noncovalently. This type of interaction is predominant in nature. For example, heparan sulfate proteoglycans in extracellular matrix bind to heparin-binding growth factors mainly through electrostatic interactions. ${ }^{3}$ Binding to heparan sulfates is critical for growth factor signaling, protection from degradation, and local accumulation of growth factors in the vicinity of cells.

Maximal dose of growth factors that can be loaded onto materials correlates with the amount of growth factor binding epitopes on the scaffolds. Designing bioactive scaffolds that can present the maximum number of epitopes while enabling control over epitope number would be beneficial for regenerative medicine applications. Several strategies for designing growth factor binding scaffolds were previously proposed. Sulfated alginate hydrogels, inspired from sulfated characteristics of glycosaminoglycans (e.g., heparan sulfate), showed superior affinity to heparin-binding growth factors relative to that of bare alginate. ${ }^{4}$ Fibrin matrices functionalized with 12-14 type three repeats of fibronectin, which nonselectively interact with various growth factors, performed as an

Received: July 13, 2012

Revised: September 10, 2012

Published: September 10, 2012 

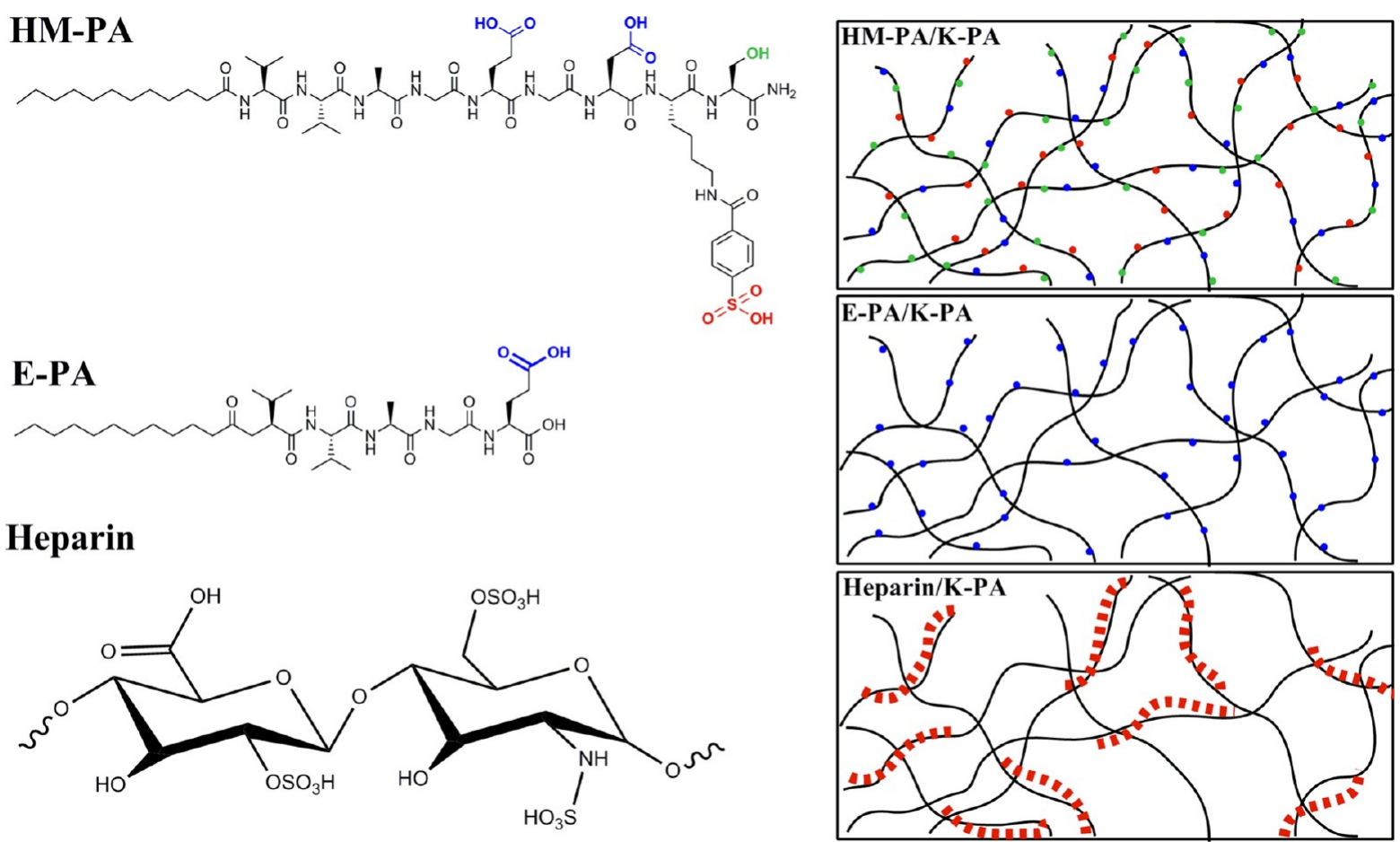

Figure 1. Nanofiber formation mechanism. Negatively charged HM-PA, E-PA, or heparin was mixed with positively charged K-PA to form respective nanofibers. Tiny spheres on nanofibers depict functional groups (red - sulfonate; green - hydroxyl; blue - carboxylate). Dashed red line indicates heparin. After nanofiber formation, heparin and functional groups of PAs are assumed to be presented by nanofibers into surrounding aqueous media. Density of heparin and functional groups of HM-PA on PA nanofibers do not reflect actual density; schematic images are used for representative purposes.

efficient growth factor delivery system, intensifying growth factor bioactivity in vitro. ${ }^{5}$ However, the relatively larger meshwork size of these polymeric scaffolds limits the density of epitope presentation. Moreover, epitope concentration on a polymer chain should be determined before synthesis. On the other hand, peptide amphiphiles, which are small building blocks, can be triggered to form supramolecular assemblies such as high-aspect-ratio nanofibers in a controlled manner. ${ }^{6}$ Nanoscale properties maximize epitope density, while epitope concentration can be controlled via epitope dilution before inducing nanofiber formation. ${ }^{7}$ For example, heparin-binding PAs formed nanofiber scaffolds when mixed with heparin, where heparin was used to bind to growth factors inside the scaffold. ${ }^{8}$ This system induced in vivo angiogenesis more efficiently than standard scaffolds. However, since long-chain heparin was used as the growth factor binding ligand, epitope dilution was limited. Moreover, contaminants in heparin batches have been reported to induce side effects in humans. ${ }^{9}$

Considering these issues, we recently designed a heparin mimetic PA (HM-PA) molecule, which bears key functional groups present in heparin and can be readily induced to form nanofiber scaffolds for regenerative medicine applications. ${ }^{10} \mathrm{We}$ have shown that these nanofibers bind vascular endothelial growth factor (VEGF) and induce in vitro and in vivo angiogenesis efficiently. The arrangement of functional groups on HM-PA/K-PA nanofibers is sufficient to bind other heparinbinding growth factors and in a manner that would elevate the bioactivity of these growth factors. In this work, we studied the interactions of heparin mimetic peptide nanofibers with various growth factors and the bioactivity of nanofiber-growth factor complexes. This study presents the high potential of heparin mimetic peptide nanofibers in binding various heparin-binding growth factors, which are widely used in regenerative medicine and in directing cellular activity.

\section{MATERIALS AND METHODS}

Materials. 9-Fluorenylmethoxycarbonyl (Fmoc) and tert-butoxycarbonyl (Boc) protected amino acids, [4-[ $\alpha$-(20,40-dimethoxyphenyl) Fmoc-aminomethyl] phenoxy] acetamidonorleucyl-MBHA resin (Rink amide MBHA resin), Fmoc-Asp $(\mathrm{OtBu})-W a n g$ resin, and 2-(1Hbenzotriazol-1-yl)-1,1,3,3-tetramethyluronium hexafluorophosphate (HBTU) were purchased from NovaBiochem and ABCR. The other chemicals for PA synthesis were purchased from Fisher, Merck, Alfa Aesar, or Aldrich. All chemicals were used as provided. Heparin and chondroitin sulfate were purchased from Sigma-Aldrich. ELISA reagents were obtained from Invitrogen. Paired antibodies for different growth factors were purchased from R\&D, except for VEGF (Invitrogen). Gold-attached secondary antibody (Aurion Immunogold reagent) was obtained from Electron Microscopy Sciences. Growth factors were obtained from e-bioscience $\left(\mathrm{VEGF}_{121}, \mathrm{HGF}\right.$, FGF-2, BMP-2), Invitrogen $\left(\right.$ VEGF $\left._{165}\right)$, and Sigma-Aldrich (NGF).

Peptide Synthesis. HM-PA and Lauryl-VVAGK-Am (K-PA) were constructed on Rink Amide MBHA resin, while Lauryl-VVAGE-Am (E-PA) was constructed on Fmoc-Asp $(\mathrm{OtBu})$-Wang resin (Figure S1, Supporting Information). Amino acid couplings were performed with 2 equiv of Fmoc-protected amino acid, 1.95 equiv of HBTU, and 3 equiv of $\mathrm{N}, \mathrm{N}$-diisopropylethylamine (DIEA) for $2 \mathrm{~h}$. To remove the Fmoc group, $20 \%$ (v/v) piperidine/dimethylformamide solution (DMF) was added for $20 \mathrm{~min}$. To block the remaining free amine groups after amino acid coupling, $10 \%(\mathrm{v} / \mathrm{v})$ acetic anhydride solution in DMF was used $(30 \mathrm{~min})$. After each step, the resin was washed by using DMF, dichloromethane (DCM), and DMF (three times each). To synthesize HM-PA, sulfobenzoic acid was added to the side chain of lysine. A lysine residue with 4-methytrityl (Mtt) side-chain protection was used for selective deprotection of amine groups. Resins were treated with a TFA/TIS $/ \mathrm{H}_{2} \mathrm{O} / \mathrm{DCM}$ mixture (5:2.5:2.5:90 ratio; $\mathrm{TFA}=$ trifluoroacetic acid; $\mathrm{TIS}=$ triisopropyl 

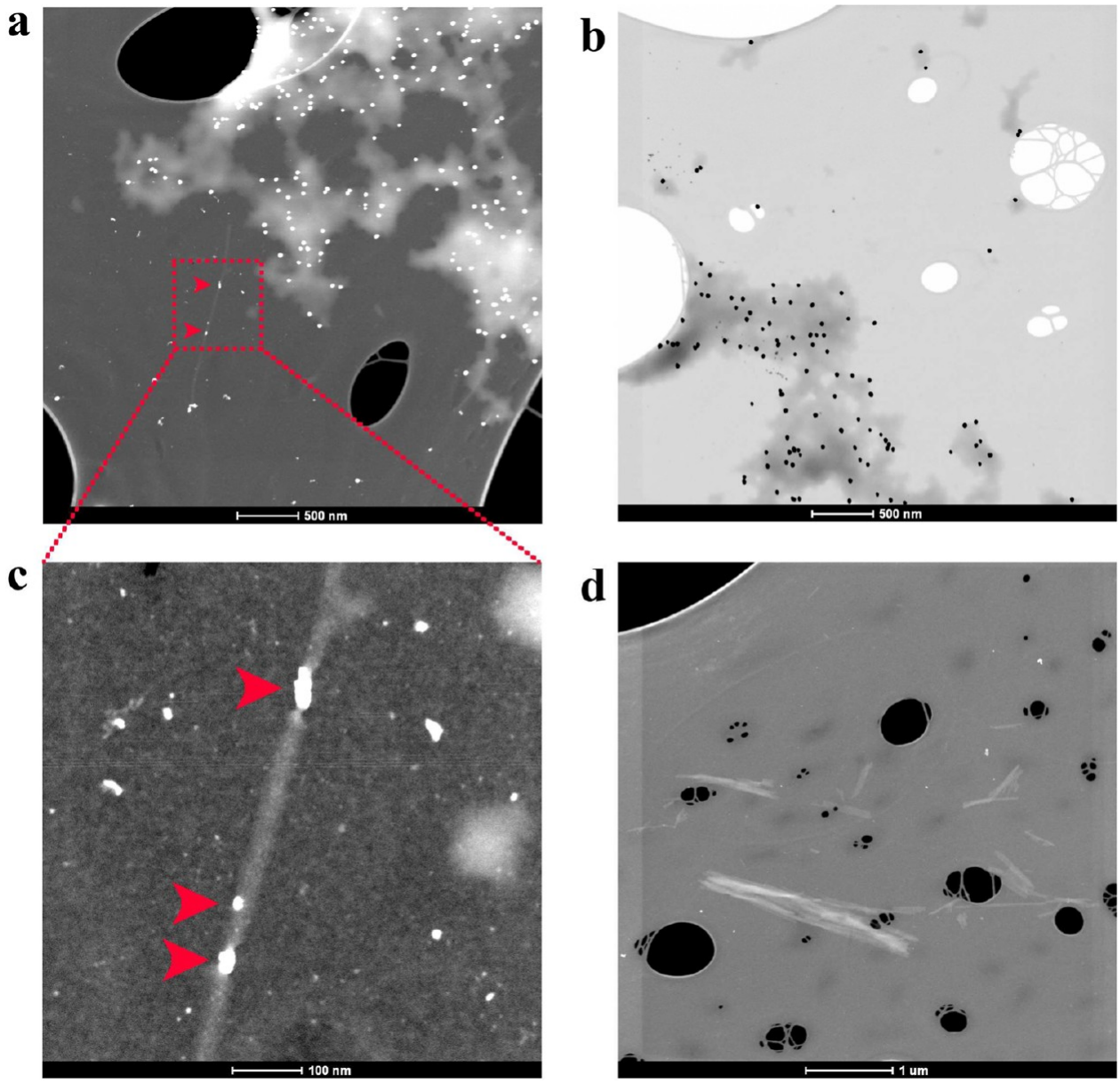

Figure 2. TEM images of immunogold stained HGF on HM-PA/K-PA nanofibers. (a) HGFs were observed on both individual nanofibers and PA aggregates. White dots indicate gold nanoparticles. (b) Inverted image, gold nanoparticles were visualized as black dots. (c) Magnified version of an individual nanofiber shown in image a, presenting gold nanoparticles. (d) Negative control (without primary antibody) shows no staining.

silane) for $5 \mathrm{~min}$ to remove Mtt. To cleave PAs from the resin, TFA/ TIS $/ \mathrm{H}_{2} \mathrm{O}$ mixture (95:2.5:2.5 ratio) was treated with resin for $2 \mathrm{~h}$. Excess TFA was removed by rotary evaporation. The remaining viscous PA solution was triturated with ice-cold ether, and the resulting white precipitate was dissolved in aqueous solution and freeze-dried. PAs were characterized by liquid chromatography and mass spectrometry (LC-MS). Mass spectrum was obtained with Agilent 1200 LC-MS equipped with Agilent 6530 Q-TOF with an ESI source and Zorbax Extend-C18 $2.1 \times 50 \mathrm{~mm}$ column for basic conditions and Zorbax SB-C8 $4.6 \mathrm{~mm} \times 100 \mathrm{~mm}$ column for acidic conditions. A gradient of (a) water $(0.1 \%(\mathrm{v} / \mathrm{v})$ formic acid or $0.1 \%$ $\left.(\mathrm{v} / \mathrm{v}) \mathrm{NH}_{4} \mathrm{OH}\right)$ and $(\mathrm{b})$ acetonitrile $(0.1 \%(\mathrm{v} / \mathrm{v})$ formic acid or $0.1 \%$ (v/v) $\mathrm{NH}_{4} \mathrm{OH}$ ) was used. An Agilent 1200 preparative reverse-phase HPLC system equipped with a Zorbax Extend-C18 $21.2 \times 150 \mathrm{~mm}$ column for basic conditions and a Zorbax SB-C8 $21.2 \times 150 \mathrm{~mm}$ column for acidic conditions was used to purify the peptides. A gradient of $(\mathrm{a})$ water $\left(0.1 \%(\mathrm{v} / \mathrm{v})\right.$ TFA or $\left.0.1 \%(\mathrm{v} / \mathrm{v}) \mathrm{NH}_{4} \mathrm{OH}\right)$ and (b) acetonitrile $\left(0.1 \%(\mathrm{v} / \mathrm{v})\right.$ TFA or $\left.0.1 \%(\mathrm{v} / \mathrm{v}) \mathrm{NH}_{4} \mathrm{OH}\right)$ was used.

Nanofiber Formation Mechanism. Nanofiber formation mechanisms were based on mixing oppositely charged PAs, which neutralized net charge on each other and induced self-assembly to higher-order nanofibers. Three different types of nanofibers were used in this study (Figure 1). Bioactive HM-PA nanofiber was prepared similarly to our previous work. ${ }^{10}$ HM-PA $(-3$ charge $)$ and K-PA (+1 charge) were mixed in a 1:2 molar ratio for HM-PA/K-PA nanofibers, while E-PA ( -2 charge) and K-PA (+1 charge) were mixed in a 1:1 molar ratio for control E-PA/K-PA nanofibers, to render both nanofibers negatively charged. Since the K-PA amount is lower for E-PA/K-PA nanofibers than HM-PA/K-PA nanofibers in this case, we used E-PA/K-PA 2x nanofibers as a control for this issue. For E-PA/KPA $2 \mathrm{x}$ nanofibers, we used both E-PA and K-PA in doubled concentrations but same molar ratio (1:1). Heparin carrying nanofibers were also prepared by mixing heparin and K-PA as in our previous work, ${ }^{10}$ while the weight ratio was $1: 2$ (heparin to K-PA) in this work. Heparin/K-PA nanofibers were used as a positive control. All PAs used for nanofiber formation were at $\mathrm{pH} 7$.

ELISA-Based Binding Assay. ELISA technique was exploited to compare binding levels of growth factors to HM-PA/K-PA nanofibers and control nanofibers (Figure 1). MaxiSorp plates (Thermo Scientific, NUNC) were coated with PA nanofibers or blank solution overnight at $4{ }^{\circ} \mathrm{C}$. The PA nanofiber formation was performed on plates by mixing negatively and positively charged PAs. Briefly, $0.05 \%$ HM-PA $/ 0.05 \%$ K-PA, $0.025 \%$ E-PA/0.025\% K-PA, $0.05 \%$ E-PA/ $0.05 \% \mathrm{~K}-\mathrm{PA}$, or $0.05 \%$ heparin/0.1\% K-PA (all are w/v) equal volume mixtures were prepared. The next day, the solutions were removed and the wells were washed with washing buffer (Tween 20 in $0.9 \%$ (w/v) $\mathrm{NaCl}$ solution, $\mathrm{pH}=7.4$ ). These plates are high-affinity binding plates for a broad range of molecules with hydrophobic/hydrophilic character, thus, even after extensive washing, plates were observed to be coated completely with PA nanofibers (Figure S3). After tapping for drying, blocking buffer was added. This was followed by the addition of growth factor solution, biotinylated antibody against growth factor, streptavidin-linked horseradish peroxidase (HRP) and HRP substrate $\left(3,3^{\prime}, 5,5^{\prime}\right.$-tetramethylbenzidine (TMB)). After $15-20$ min of incubation, the reaction was stopped with sulfuric acid. Absorbance change due to color formation was measured by a Spectramax M5 microplate reader (Molecular Devices) at $450 \mathrm{~nm}$ wavelength. This value was subtracted from the reference value (650 
Table 1. Growth Factors Used in This Study

\begin{tabular}{|c|c|c|c|}
\hline growth factor & physiological function $^{a}$ & $\begin{array}{l}\text { heparin } \\
\text { affinity }\end{array}$ & $\begin{array}{c}\text { dissociation } \\
\text { constant }\left(K_{\mathrm{d}}\right)^{b}\end{array}$ \\
\hline $\begin{array}{l}\text { vascular endothelial growth factor } \\
\text { (VEGF) }\end{array}$ & angiogenesis, vasculogenesis, endothelial cell growth and migration & $\begin{array}{c}\text { high } \\
\left(\text { VEGF }_{165}\right) \\
\text { none } \\
\left(\text { VEGF }_{121}\right)\end{array}$ & $165 \mathrm{nM}^{30}$ \\
\hline hepatocyte growth factor (HGF) & cell motility, mitogenesis and matrix invasion & high & $12 \mathrm{nM}^{30}$ \\
\hline fibroblast growth factor-2 (FGF-2) & $\begin{array}{l}\text { migration, proliferation and survival of endothelial cells, inhibition of differentiation of } \\
\text { embryonic stem cells }\end{array}$ & high & $\begin{array}{l}23 \mathrm{nM}^{30} \\
39 \mathrm{nM}^{31}\end{array}$ \\
\hline $\begin{array}{l}\text { bone morphogenetic protein-2 } \\
(\text { BMP-2) }\end{array}$ & differentiation and migration of osteoblasts & high & $20 \mathrm{nM}^{17}$ \\
\hline nerve growth factor (NGF) & survival and differentiation of neural cells & moderate & not determined \\
\hline
\end{tabular}

${ }^{a}$ Functions were reproduced from Lee et al. ${ }^{2}$ and NCBI. ${ }^{b}$ Dissociation constant of heparin-growth factor interaction.

$\mathrm{nm})$. All treatments were performed with three replicates and are shown as mean \pm standard deviation. Experiments were repeated at least two times independently. For statistical analysis, two-way ANOVA with Bonferroni posthoc analysis (Figure 2a) and Student's $t$ test (Figure $2 \mathrm{~b}, \mathrm{c}$ ) were used.

Atomic Force Microscopy (AFM) Imaging. AFM imaging was performed to determine the coating efficiency of PA nanofibers on ELISA plates. Coating was performed similarly to "ELISA-binding assay" procedures. After overnight incubation of plates with PA nanofiber solution, the solution was aspirated and the plate was washed 2-3 times with ELISA washing buffer (Tween 20 in $0.9 \%$ (w/ v) $\mathrm{NaCl}$ solution, $\mathrm{pH}=7.4$ ). The plates were dried by tapping, and the coated bottom part was removed for AFM imaging. Bare ELISA plates were also imaged with AFM to understand their surface roughness. Non-contact-mode AFM was performed by using model MFP-30 from Asylum Research. All images were taken with a $0.5 \mathrm{~Hz}$ scan rate. Tips with resonance frequency of $300 \mathrm{kHz}$ and spring constant of $40 \mathrm{~N} / \mathrm{m}$ were used in all experiments (BudgetSensors).

Isothermal Titration Calorimetry (ITC). To investigate the interaction between HM-PA/K-PA nanofibers and VEGF121, we used the iTC200 system (MicroCal, GE Healthcare). VEGF $_{121}(0.27 \mathrm{mg} /$ $\mathrm{mL}$ in $1 \mathrm{x}$ PBS solution) was titrated into HM-PA/K-PA nanofiber solution. For HM-PA/K-PA nanofiber solution, 0.04\% HM-PA and $0.04 \% \mathrm{~K}-\mathrm{PA}$ (both in $\mathrm{H}_{2} \mathrm{O}$ and $\mathrm{w} / \mathrm{v}$ ) were mixed to form nanofibers in solution, which was diluted 2-fold with $2 \mathrm{x}$ PBS to obtain HM-PA/KPA nanofiber solution in 1x PBS. Reaction was performed at $25{ }^{\circ} \mathrm{C}$ with $500 \mathrm{rpm}$ stirring speed. Twenty injections were performed, where the injection period was $4 \mathrm{~s}$ and the space between injections was 150 s. All solutions that were used in ITC experiments were at $\mathrm{pH} 7$.

Immunogold Staining and Transmission Electron Microscopy (TEM) Imaging. Hepatocyte growth factor (HGF) binding on HM-PA/K-PA nanofibers was visualized by using immunogold staining and TEM imaging. First, HM-PA/K-PA gel was formed by mixing $20 \mu \mathrm{L}$ of $1 \%$ HM-PA with $1 \%$ K-PA (w/v). Gel was diluted 10 times, and $30 \mu \mathrm{L}$ of the diluted solutions was dropped onto paraffin film. $\mathrm{Cu}$ grids were reversed onto these drops and incubated for $5 \mathrm{~min}$. The tiny amount of liquid left on the grids was absorbed with dust-free paper, and the grids were dipped into $1 \mathrm{x}$ PBS solution two times to wash weakly bound nanofibers. Grids were reversed onto $30 \mu \mathrm{L}$ of blocking solution (Assay buffer, Invitrogen) and incubated for $1 \mathrm{~h}$ at room temperature. Drops on grids were absorbed, and HGF (500 ng/ $\mathrm{mL}$ in assay buffer) was added onto the grids. After $2 \mathrm{~h}$ of incubation, grids were washed with PBS five times. Primary antibody $(25 \mu \mathrm{g} / \mathrm{mL}$, R\&D) against human HGF was added onto grids and incubated overnight at $4{ }^{\circ} \mathrm{C}$. Grids were washed with PBS 5 times. Gold-attached antibody ( $25 \mathrm{~nm}$ gold particles conjugated to antimouse $\operatorname{IgG}$ ), 1/20 diluted from stock with assay buffer, was put onto paraffin film, and grids were reversed onto this solution, to prevent precipitation of gold particles onto the grid surface due to gravitation. After $1 \mathrm{~h}$, grids were washed five times with PBS and three times with double-distilled water $\left(\mathrm{ddH}_{2} \mathrm{O}\right)$. After drying at room temperature for at least $3 \mathrm{~h}$, TEM (FEI, Tecnai G2 F30) imaging was performed. All images were taken in STEM mode with an HAADF (high angle annular dark field) detector.
HM-PA/K-PA Nanofiber versus Heparin Competition Assay. HM-PA/K-PA nanofiber and heparin competition for the same site of growth factor was studied by increasing doses of heparin $(0.0003 \%$ to $0.3 \%, \mathrm{w} / \mathrm{v}$ ) in the presence of various growth factors just before latter ones were incubated with HM-PA/K-PA nanofibers. HM-PA/K-PA nanofiber coating and the rest of the assay was performed similarly to ELISA-based binding assay. $\mathrm{IC}_{50}$ (half-maximal inhibitory concentration) of heparin to inhibit growth factor and HM-PA/K-PA nanofiber binding was calculated for each growth factor by using GraphPad Prism software. Nonlinear regression analysis with robust fitting was carried out for this purpose. We performed the same experiment with chondroitin sulfate instead of heparin (only for fibroblast growth factor-2 (FGF-2)) to understand the effect of heparin. All treatments were performed with three replicates and are shown as mean \pm standard deviation. Experiments were repeated twice, independently.

NGF Induced Neurite Extension Assay. To determine biological activity caused by interaction of PA nanofibers with nerve growth factor (NGF), PC-12 cells were cultured on NGF-coated PA nanofiber surfaces. PA nanofiber gel scaffolds with -1 charge were prepared as described below. For HM-PA/K-PA gel, $1.5 \mathrm{mM}$ HM-PA was mixed with $3 \mathrm{mM} \mathrm{K}-\mathrm{PA}$ at equal volumes ( $40 \mu \mathrm{L}$ for 96 well-plate). E-PA/KPA gel was formed in the same way by mixing $1.5 \mathrm{mM}$ E-PA with 1.5 $\mathrm{mM}$ K-PA while E-PA/K-PA $2 \mathrm{x}$ gel was prepared by mixing $3 \mathrm{mM}$ EPA with $3 \mathrm{mM}$ K-PA. $0.1 \%(\mathrm{w} / \mathrm{v})$ Poly-D-lysine (PDL) was coated as a control since it is not expected to bind NGF due to its dense positive charge. After gel formation, plates were dried under a laminar flow hood and UV sterilized. For NGF coating on PA coated surfaces, NGF was added at concentrations of $10 \mathrm{ng} / \mathrm{mL}$ or $50 \mathrm{ng} / \mathrm{mL}$. After $2 \mathrm{~h}$ of incubation at room temperature, wells were washed three times with PBS to remove any unbound NGF. PC- 12 cells $\left(5 \times 10^{3}\right.$ cells/well $)$ were then seeded and cultured on these surfaces for 4 days. In another experimental group, incubated NGF was not removed to test the effect of surface bound and soluble NGF at the same time (total concentration was the same with other groups). In this case, cells were added directly on NGF solution over PA or PDL coated surfaces. For soluble NGF groups, the same concentration of NGF was added on cells after seeding ( $n=3$ for all samples). At the end of 4 days, images ( 5 images/well) were taken at $200 \mathrm{x}$ magnification, and neurite lengths were quantified with Image J. For statistical analysis, two-way ANOVA with Bonferroni posthoc analysis was performed (Figures 6 and S4).

For immunostaining, PA nanofiber coating was carried out by using the same protocol except that PAs were coated on glass coverslips placed in 24 well-plates (total volume: $300 \mu \mathrm{L} /$ well). NGF coating (50 $\mathrm{ng} / \mathrm{mL}$ ) was performed as described above and $3 \times 10^{4}$ cells/well were seeded. After 4 days of culture, cells were fixed with $4 \%$ paraformaldehyde, permeabilized with $0.3 \%$ TritonX-100, and blocked with $10 \%$ goat serum. Antibodies against $\beta$-III-Tubulin (Millipore, 1:250 dilution) and synaptophysin 1 (Sigma-Aldrich, 1:400 dilution) were incubated overnight at $4{ }^{\circ} \mathrm{C}$. After washing, cells were incubated with goat-antirabbit IgG-Cy3 (Chemicon, 1:200 dilution) and goatantimouse IgG-Cy2 (Chemicon, 1:400 dilution) for $1 \mathrm{~h}$ at room temperature and washed to remove any unbound antibodies. 
Coverslips were then removed from wells, mounted by using Prolong gold antifade reagent (Invitrogen), and imaged with a confocal microscope (Carl Zeiss, LSM510).

\section{RESULTS AND DISCUSSION}

3.1. HM-PA Nanofibers Bind Heparin-Binding Growth Factors with Strong Affinity. Heparin-binding growth factors that are widely used in regenerative medicine studies were studied for testing the efficacy of growth factor-binding peptide nanofibers (Table 1). Peptide molecules forming the nanofibers are depicted in Figure 1, and nanofiber formation mechanisms are explained in the Materials and Methods section. While forming nanofibers, the ratio of negatively charged PAs (HM-PA and E-PA) to positively charged K-PA in nanofibers is critical as it determines the net charge on nanofibers. Ratios of 1:2 and 1:1 for HM-PA/K-PA and E-PA/ $\mathrm{K}-\mathrm{PA}$ were chosen, respectively, in order to render both nanofibers net one negative charge. The E-PA cannot retain its negative charge at a higher ratio of K-PA (1:2) and loses its capacity to hold growth factors (data not shown).

To validate and visualize growth factor-peptide nanofiber interactions, we performed immunogold staining of growth factors bound to the nanofibers. For this experiment, we chose HGF as the model growth factor. TEM was used to image the complex of peptide nanofibers and gold nanoparticleconjugated antibody against HGF. Both individual nanofibers (Figure 2a,c) and nanofiber aggregates (Figure 2a,b) were observed to bind growth factors (white dots in a,c; black dots in b). When primary antibody for growth factor was not used, no growth factor binding was observed, excluding the possibility of nonspecific binding of gold attached secondary antibodies to nanofibers (Figure 2d). Nanofiber aggregates bound to growth factors in TEM imaging were studied by energy-dispersive $\mathrm{X}$ ray (EDX) analysis, and a strong sulfur signal caused by sulfonate group of HM-PA (Figure S2) was observed demonstrating the presence of peptide nanofibers interacting with the growth factor.

Growth factor-peptide nanofiber interaction was further investigated in-depth to understand the effect of heparin mimicking functional groups (carboxylate, hydroxyl, and sulfonate) on nanofibers in growth factor binding. ELISAbased growth factor binding assay was used to study the binding mechanism. All three types of peptide nanofibers were coated efficiently onto an ELISA plate as revealed by AFM imaging, which were clearly distinguishable in terms of roughness compared to bare ELISA plate (Figure S3). After confirming the nanofiber coating on ELISA plates, growth factors bound to the peptide nanofibers were detected with ELISA assay.

HM-PA nanofibers were designed to mimic heparin and showed a higher binding level to three growth factors-HGF, VEGF, and FGF-2-than control E-PA nanofibers. This difference confirms that growth factor and nanofiber interaction is further strengthened by additional functional groups on HMPA/K-PA nanofibers (Figure 3). Heparin-carrying (heparin with K-PA) nanofibers, which were included as a positive control, showed the strongest binding signal (Figure 3a). Meanwhile, bare plate surface, which was the negative control, showed the weakest signal (shown for $\mathrm{VEGF}_{165}$ in Figure 3a and other growth factors in Figure S4). HM-PA/K-PA nanofibers showed a comparable level of binding to $\mathrm{VEGF}_{165}$ with heparin-carrying nanofibers (Figure 3a). Nanofiber control (E-PA/K-PA) showed significantly less binding level to a

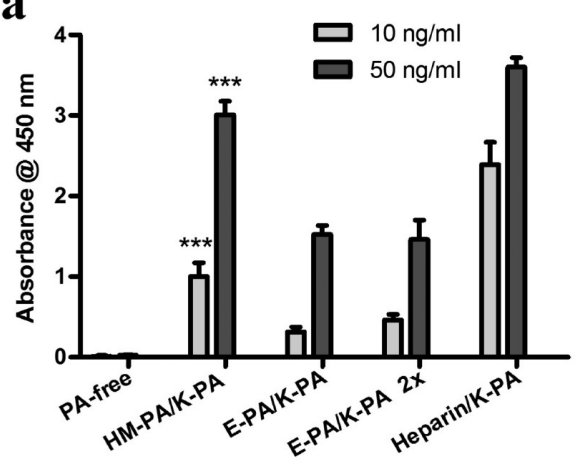

b

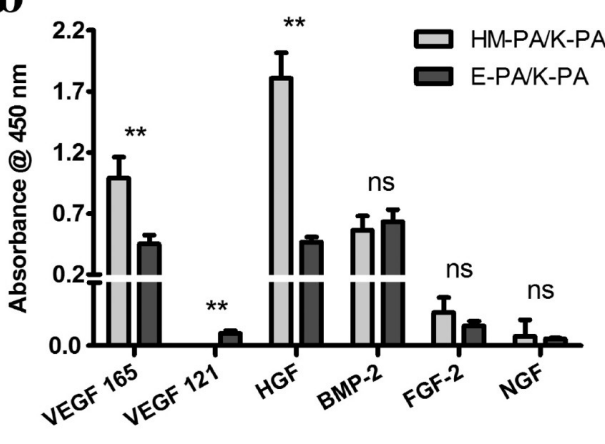

c

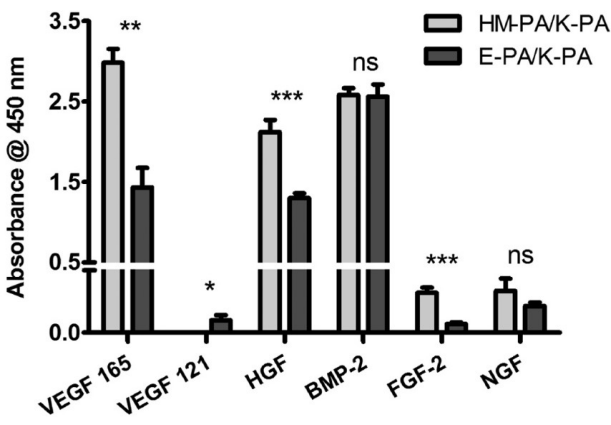

Figure 3. ELISA-based binding assay. (a) Binding levels of $10 \mathrm{ng} / \mathrm{mL}$ or $50 \mathrm{ng} / \mathrm{mL}$ of VEGF $_{165}$ with different nanofibers or PA-free surface are shown. The difference between HM-PA/K-PA and other treatments was significant for both growth factor concentrations. (b) Binding levels of different growth factors $(10 \mathrm{ng} / \mathrm{mL})$ to HM-PA/KPA or E-PA/K-PA nanofibers are compared. (c) Binding levels of different growth factors $(50 \mathrm{ng} / \mathrm{mL})$ to HM-PA/K-PA and E-PA/KPA nanofibers are compared. $* * * p<0.001, * * p<0.01, * p<0.05$, ns $=$ not significant. Statistical analysis was performed with two-way ANOVA with Bonferroni posthoc analysis (a) and Student's $t$ test (b and c). Experiments were performed as three replicates $(n=3)$.

VEGF $_{165}$ compared to HM-PA/K-PA nanofibers. An E-PA/KPA 2x sample containing two-folds higher control nanofiber concentration was used to eliminate the effect of incomplete coverage of the surface on binding. There was no significant difference between E-PA/K-PA and E-PA/K-PA 2x samples, indicating complete coverage and saturation of the surface with one-fold control nanofibers (Figure 3a).

Growth factor-peptide nanofiber interactions were analyzed at two different growth factor concentrations to investigate the effect of growth factor concentration on nanofiber binding. VEGF $_{165}$ and HGF showed higher level of binding to HM-PA/ K-PA nanofibers than E-PA/K-PA nanofibers for both $10 \mathrm{ng} /$ $\mathrm{mL}$ and $50 \mathrm{ng} / \mathrm{mL}$ growth factor concentrations (Figure 2b,c). In addition, FGF-2 bound to HM-PA/K-PA nanofibers 
significantly more than control nanofibers at higher growth factor concentration (Figure 2c). In spite of its heparin-binding property, BMP-2 exhibited a strong binding pattern to both HM-PA/K-PA and control nanofibers at both low and high growth factor concentrations (Figures $2 \mathrm{~b}$ and $2 \mathrm{c}$ ). Negative charge provided by carboxylate groups on control peptide nanofibers might be sufficient to bind to BMP-2 strongly, leading to similar binding pattern of BMP-2 to E-PA/K-PA and HM-PA/K-PA nanofibers. However, the binding site of BMP-2 might differ between HM-PA/K-PA and E-PA/K-PA nanofibers, which could change its bioactivity.

One of the weakest binding patterns was observed with NGF, where HM-PA/K-PA nanofibers showed slightly better binding at higher growth factor concentration. However, it was clear that even this degree of binding makes a remarkable difference in inducing cellular activity, which will be described in more detail in the following sections (Figures 5-7).

VEGF $_{121}$, which lacks heparin-binding domain, did not reveal any binding signal to HM-PA/K-PA nanofibers, while it exhibited very weak binding to E-PA/K-PA nanofibers. Thus, heparin-binding domain of VEGF is critical for its binding to HM-PA/K-PA nanofibers. These results indicate that HM-PA/ K-PA nanofibers with heparin mimicking functional groups stand to be an excellent analogue for heparin regarding growth factor binding capability.

3.2. Role of Heparin-Binding Domains of Growth Factors in Their Interaction with HM-PA/K-PA Nanofibers. While growth factor-nanofiber binding has been emphasized in the literature, the binding site of growth factor to material should also be taken into consideration when designing biomaterials for regenerative medicine applications. Growth factors interact with heparan sulfates through their "heparin-binding domain". ${ }^{11-17}$ Growth factor-heparan sulfate binding induces dimerization/oligomerization of growth factor receptors (generally tyrosine kinase receptors), which is required for autophosphorylation of receptors and subsequent activation of signaling pathways. ${ }^{18-20}$ For signaling to be effective, a threshold number of receptor-growth factor complexes should be active on the surface of cells for an appropriate period of time. ${ }^{21}$ This requires stability of growth factor-receptor interactions, which are maintained by heparan sulfates acting as coreceptors. ${ }^{21,22}$ Thus, a material designed to mimic heparan sulfates should bind to growth factors through their heparin-binding domains, which would prevent blocking of other sites on growth factors such as "receptor binding site" that is critical for signaling.

$\mathrm{VEGF}_{165}$ and $\mathrm{VEGF}_{121}$ exhibited significantly different binding levels to HM-PA/K-PA nanofibers in ELISA-based binding assay. To clarify the necessity of heparin-binding domain in HM-PA/K-PA nanofiber - growth factor interaction, differential affinity of $\mathrm{VEGF}_{165}$ and $\mathrm{VEGF}_{121}$ to HM-PA/K-PA nanofibers was further tested by using ITC. Previously, binding constant between $\mathrm{VEGF}_{165}$ and HM-PA/K-PA nanofibers was measured by using this technique. ${ }^{10}$ Here, interaction between VEGF $_{121}$ and HM-PA/K-PA nanofibers was investigated through a similar protocol that we used in our previous study. ${ }^{10}$ While the binding constant of HM-PA/K-PA$\mathrm{VEGF}_{165}$ was similar to the binding constant of heparinVEGF $_{165},{ }^{10} \mathrm{VEGF}_{121}$ revealed no binding signal with HM-PA/ K-PA, further supporting the critical contribution of the heparin-binding domain in VEGF-HM-PA/K-PA nanofiber interaction (Figure S5).
To understand the role of heparin-binding domains of other growth factors in HM-PA/K-PA nanofiber binding, a competition assay was performed, where heparin and HMPA/K-PA nanofibers competed for binding to growth factors. In this assay, growth factors bound to heparin were expected to be washed away, while those that bound to HM-PA nanofibers were expected to stay in the plate and be detected with ELISA. Thus, signal diminution in ELISA would indicate that both heparin and HM-PA/K-PA nanofibers compete for the same binding site. In total agreement with previous assays, the VEGF $_{165}$ binding signal showed a very sharp decline as heparin concentration was increased, further supporting that HM-PA/ K-PA nanofibers compete for nonredundant heparin-binding domain (Figure 4a). Interestingly, only FGF-2 showed a similar
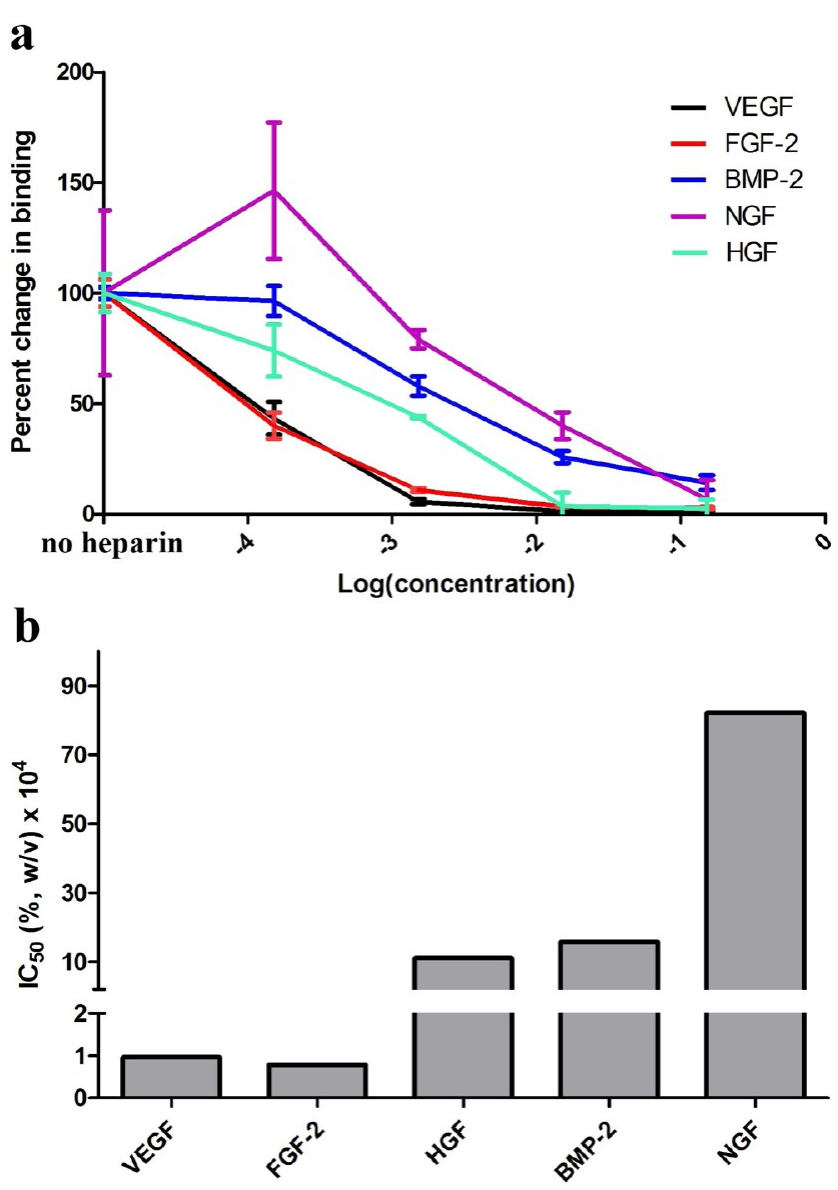

Figure 4. Competition assay between heparin and HM-PA/K-PA nanofibers for growth factor binding. (a) Dose-dependent interference of heparin with growth factor binding to HM-PA/K-PA nanofibers. For each growth factor, the binding signal to HM-PA/K-PA nanofibers when there was no heparin in milieu was taken as $100 \%$. (b) $\mathrm{IC}_{50}$ value of heparin for inhibiting HM-PA/K-PA nanofiber binding of each growth factor was calculated and represented. Experiment was performed as three replicates $(n=3)$.

pattern to $\mathrm{VEGF}_{165}$ among other growth factors (Figure 4). The inhibitory effect was specific to heparin, since chondroitin sulfate (another sulfated glycosaminoglycan) was not able to inhibit FGF-2 binding to HM-PA/K-PA nanofibers (exhibited only limited inhibitory effect at maximal dose, Figure S6). Heparin was more inhibitory at its minimal dose than chondroitin sulfate at its maximal dose. Since heparan sulfate (or heparin) binding is critical for bioactivity of $\mathrm{VEGF}_{165}$ and 

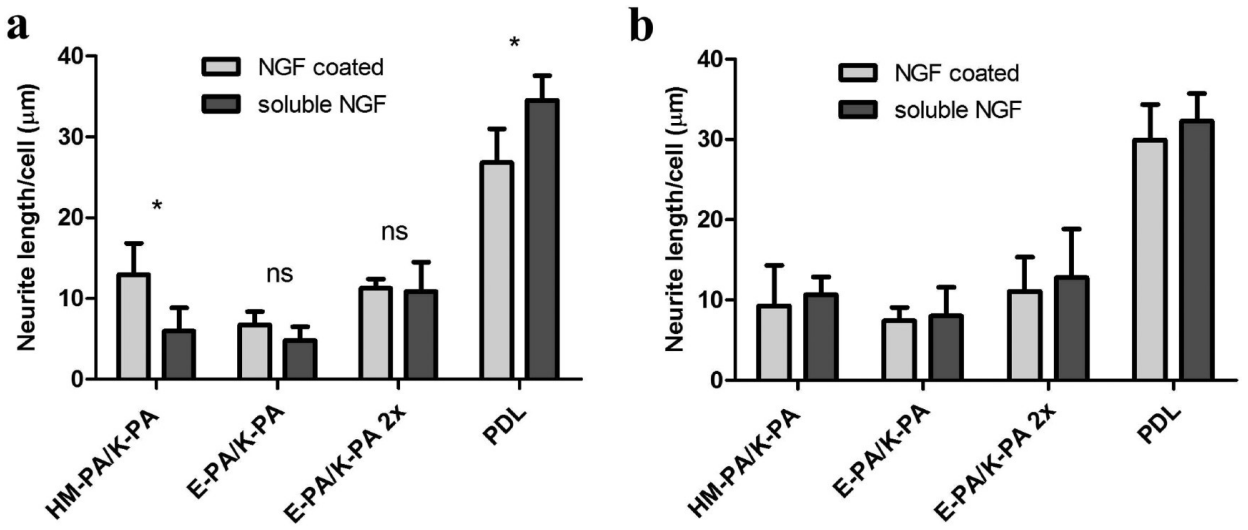

Figure 5. Results of neurite outgrowth assay on NGF treated substrates and NGF-free substrates. NGF coated in the above figure indicates that substrates were treated with NGF without removal of unbound NGF on which cells were seeded. Soluble NGF indicates that cells cultured on NGFfree substrates were induced with soluble NGF in culture media. NGF amounts used were $10 \mathrm{ng} / \mathrm{mL}$ (a) or $50 \mathrm{ng} / \mathrm{mL}$ (b). There was no significant difference for any substrate when coated NGF was compared with soluble NGF, except for HM-PA/K-PA and PDL at $10 \mathrm{ng} / \mathrm{mL}(* p<0.05$. Statistical analysis was performed by using two-way ANOVA with Bonferroni posthoc analysis, $n=3$ ).

FGF-2, ${ }^{23,24}$ binding to the same site with heparin renders HMPA/K-PA nanofibers a very efficient scaffold for delivery of these growth factors. Heparin-binding domains of HGF and BMP-2 were more redundant than those of VEGF and FGF-2 in HM-PA/K-PA nanofiber binding (Figure 4a). IC I $_{50}$ for HGF and BMP-2 calculated from competitive binding curves in Figure 4a were nearly 10 times higher than $\mathrm{IC}_{50}$ of VEGF and FGF-2 (Figure 4b). Dissociation constants of heparin and these growth factors were shown to be similar to each other in the literature (Table 1). Thus, the difference between $\mathrm{IC}_{50}$ values of heparin between these two groups of growth factors cannot be attributed to any difference in their affinity to heparin. This difference could be caused by the existence of extra binding sites, other than the heparin-binding site, on BMP-2 and HGF for HM-PA/K-PA nanofiber binding. NGF binding to heparin is known to be moderate, ${ }^{25}$ compared to strong binding affinities of other growth factors, which may be the reason for poor competition between heparin and HM-PA/K-PA nanofibers for binding to NGF. Nonetheless, NGF seems to have different binding sites for HM-PA/K-PA nanofibers and heparin (Figure 4). $\mathrm{IC}_{50}$ of heparin for NGF was nearly 80 times higher than $\mathrm{IC}_{50}$ of heparin for VEGF/FGF-2 and 7-8 times higher than $\mathrm{IC}_{50}$ of heparin for HGF/BMP-2 (Figure $4 \mathrm{~b}$ ).

3.3. HM-PA/K-PA nanofiber - growth factor interaction is translated to cellular activity. As mentioned above, improper interaction of materials with growth factors (e.g., through improper binding site) can block growth factor activity unexpectedly. Hence, growth factor's biological functionality should be validated after it is tethered on the material. NGF, which had the weakest interaction among the growth factors that were tested in this study and whose heparin-binding domain was least required for HM-PA/K-PA nanofiber binding, was selected for studying biological activity. To investigate the effect of HM-PA/K-PA nanofiber binding on NGF functionality, neurite outgrowth by PC-12 cells cultured on NGF-coated PA nanofiber surfaces was analyzed. NGF retained its ability to induce neurite outgrowth fully when it was bound to HM-PA/K-PA nanofibers. Neurite outgrowth performances of PC-12 cells did not deteriorate when NGF was presented to cells as coated on nanofibers compared to its soluble form (Figure 5). To exclude any possibility of bioactivity reduction due to differential amount of growth factors between "soluble" and "coated" treatments, unbound growth factors in "coated" treatment were not removed and stayed in solution. Considering that the total growth factor amount was same in both "soluble" and "coated" treatments, HM-PA/K-PA nanofibers enhanced NGF signaling, since neurite outgrowth activity in "coated" samples was significantly higher than "soluble" samples (Figure 5a) in the presence of low growth factor concentration. No significant increase in bioactivity was observed at higher growth factor concentration (Figure $5 b$ ). This difference could be due to the fact that higher concentration of growth factors in the vicinity of cells did not necessitate their accumulation and preservation with HM-PA/ K-PA nanofibers for cellular activity. Interestingly, we did not observe such a difference with E-PA/K-PA nanofibers, indicating contribution of functional groups on HM-PA/K-PA nanofibers for NGF signaling. Moreover, higher binding of HM-PA/K-PA nanofibers to NGF, although it was statistically insignificant, was translated to elevated cellular activity when compared to control nanofiber systems (Figure 6). To test this, unbound NGFs on HM-PA/K-PA nanofiber and control surfaces were washed away, and bioactivity comparison was performed only between bound NGFs. NGF-coated and washed HM-PA/K-PA nanofibers $(50 \mathrm{ng} / \mathrm{mL})$ lead to significantly longer neurites compared to E-PA/K-PA nanofiber and PDL controls (Figure 6). There was no such difference when $10 \mathrm{ng} / \mathrm{mL}$ NGF was used, which is in good correlation with the results of growth factor binding analysis by ELISA (Figure 6). In summary, HM-PA/K-PA nanofibers interact with NGF moderately and this interaction is translated into cellular response without any loss in bioactivity of growth factor.

Promotion of the neurite length on HM-PA/K-PA nanofibers is clearly dependent on HM-PA-NGF interaction that could be translated to differentiation response upon induction of cells by NGF activity. Immunostaining against $\beta$-III-tubulin, a neuron-abundant microtubule protein, and synaptic protein Synaptophysin 1 (Syn1) revealed higher expression of these proteins when cells were cultured on HM-PA-NGF substrates (Figure 7). Neural morphology was also more prominent on this surface correlating with the longer neurites (Figure 6). $\beta$ III-tubulin is a neuron-specific tubulin subunit that is abundantly expressed along neurites as well as cell soma. ${ }^{26}$ Syn1 is a synaptic protein abundant in presynaptic nerve terminal, and its presence along the axonal protrusions indicates presynaptic nerve terminal development. ${ }^{27,28} \mathrm{Ex}-$ 


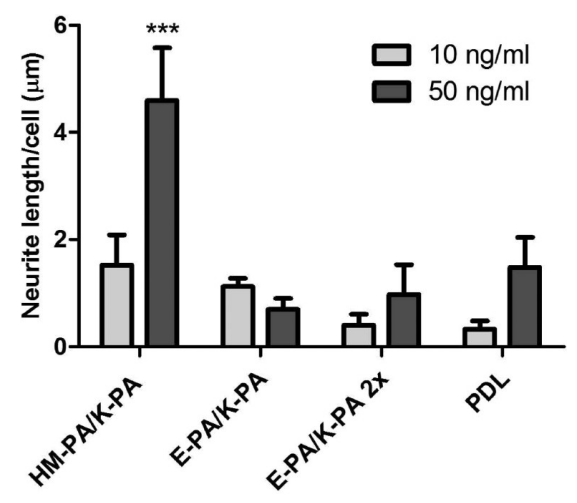

Figure 6. Results of neurite outgrowth assay on NGF-treated substrates. PDL and PA substrates were treated with $10 \mathrm{ng} / \mathrm{mL}$ or $50 \mathrm{ng} / \mathrm{mL}$ NGF after which they were washed to remove any unbound NGF. HM-PA/K-PA interaction with NGF led to longer neurites on this surface when $50 \mathrm{ng} / \mathrm{mL} \mathrm{NGF}$ was used, probably due to higher amount of NGF remaining on the HM-PA/K-PA nanofiber-coated surface than E-PA/K-PA and PDL after washing $(* * * p<0.001$ between HM-PA/K-PA and other surfaces at $50 \mathrm{ng} / \mathrm{mL}, * p<0.05$ between HM-PA/K-PA and E-PA/K-PA $2 \mathrm{x}$ or PDL, $p>0.05$ between HM-PA/K-PA and E-PA/K-PA at $10 \mathrm{ng} / \mathrm{mL}$ ). Statistical analysis was performed by using two-way ANOVA with Bonferroni posthoc analysis $(n=3)$. pression profiles of PC-12 cells cultured on HM-PA-NGF substrate is consistent with the expected localization of these neural marker proteins. $\beta$-III-tubulin was found to be localized in cell soma along with neurites (Figure 7a). Cells also expressed $\beta$-III-tubulin on E-PA/K-PA and PDL in cell soma and in a few short neurites observed on E-PA/K-PA (Figure $7 \mathrm{~d}, \mathrm{~g})$. A dramatic difference in expression profile of Syn 1 was found when cells were cultured on different substrates, which can be attributed to differential interaction of these surfaces with NGF leading to differential cell responses (Figure $7 \mathrm{~b}, \mathrm{e}, \mathrm{h}$ ). Syn 1 expression was heavily concentrated along neurites and nerve terminals on HM-PA/K-PA, while weak expression in cell soma was observed on other substrates. Thus, it can be concluded that higher level of binding of HM-PA to NGF induces neural differentiation of PC-12 cells more efficiently, leading to the formation of presynaptic nerve terminals, an indicator of neural maturation, on this substrate.

\section{CONCLUSION}

In summary, the functionality of heparan sulfates in extracellular matrix can be achieved synthetically through presenting key functional groups of heparan sulfates on peptide nanofibers. While forming hydrogel scaffolds, these nanofibers bind to heparin-binding growth factors that are utilized commonly in regenerative medicine studies and present them

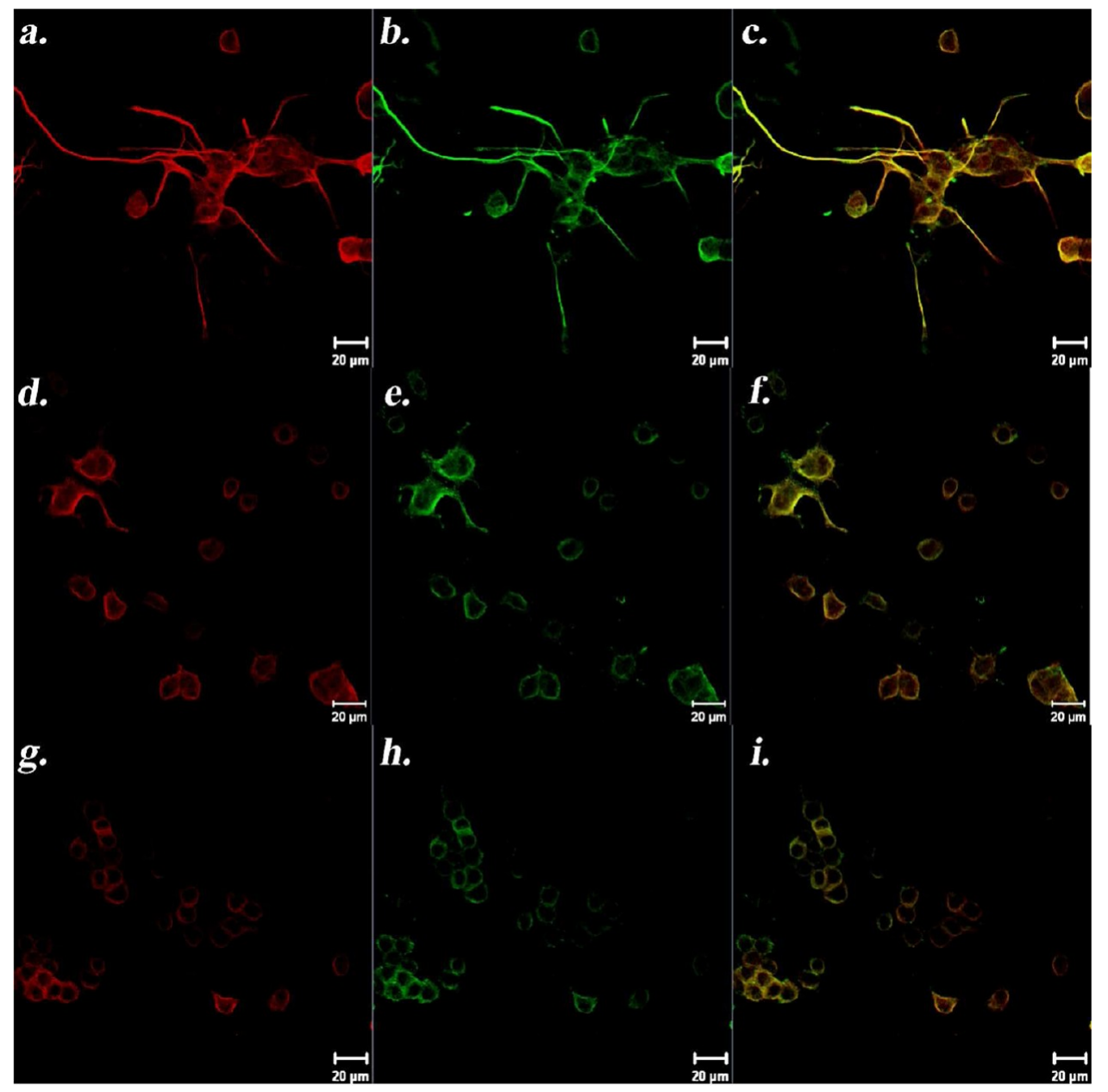

Figure 7. Immunostaining of PC-12 cells against $\beta$-III-Tubulin (a,d,g) and Syn 1 (b,e,h) on NGF treated surfaces: (a-c) HM-PA/K-PA, (d-f) EPA/K-PA, (g-i) PDL. Panels c, f and i show merged images of Synl staining and $\beta$-III-Tubulin on the same cells. Higher expression of both neural markers along with specific localization of Synaptophysin I in nerve terminals was clear in cells cultured on HM-PA/K-PA. 
to cells effectively. Interestingly, for VEGF and FGF-2, this binding specifically requires the presence of heparin-binding domain of growth factors, which may be critical for proper presentation of growth factors to cells. While we recently reported angiogenesis and neural tissue engineering applications of these scaffolds, ${ }^{10,29}$ further work is underway for other applications such as wound healing and bone and cartilage tissue engineering.

\section{ASSOCIATED CONTENT}

\section{S Supporting Information}

Additional AFM, ITC, and ELISA assay results. This material is available free of charge via the Internet at http://pubs.acs.org.

\section{AUTHOR INFORMATION}

\section{Corresponding Author}

*Mailing Address: Institute of Materials Science and Nanotechnology, National Nanotechnology Research Center (UNAM), Bilkent University, Ankara, TURKEY, 06800. Email: atekinay@unam.bilkent.edu.tr (A.B.T.) and moguler@ unam.bilkent.edu.tr (M.O.G.). Tel: +90 3122903572 (A.B.T.) and +90 3122903552 (M.O.G.). Fax: +90 3122664365 .

Notes

The authors declare no competing financial interest.

\section{ACKNOWLEDGMENTS}

We would like to thank Z. Erdogan and Z. E. Ulger for their technical help. This work was funded partially by Loreál Young Women Investigator Award and TUBITAK Grants 111M410 and 110M355. R.M. is supported by the TUBITAK-BIDEB 2215 Ph.D. fellowship. M.O.G. acknowledges support from the Turkish Academy of Sciences Distinguished Young Scientist Award (TUBA-GEBIP).

\section{REFERENCES}

(1) Mieszawska, A. J.; Kaplan, D. L. BMC Biol. 2010, 8, 59-61.

(2) Lee, K.; Silva, E. A.; Mooney, D. J. J. R. Soc., Interface 2011, 8, 153-170.

(3) Dvir, T.; Timko, B. P.; Kohane, D. S.; Langer, R. Nat. Nanotechnol. 2011, 6, 13-22.

(4) Freeman, I.; Kedem, A.; Cohen, S. Biomaterials 2008, 29, 32603268.

(5) Martino, M. M.; Hubbell, J. A. FASEB J. 2010, 24, 4711-4721.

(6) Hartgerink, J. D.; Beniash, E.; Stupp, S. I. Science 2001, 294, 1684-1688.

(7) Shah, R. N.; Shah, N. A.; Del Rosario Lim, M. M.; Hsieh, C.; Nuber, G.; Stupp, S. I. Proc. Natl. Acad. Sci. U.S.A. 2010, 107, 32933298.

(8) Rajangam, K.; Behanna, H. A.; Hui, M. J.; Han, X.; Hulvat, J. F.; Lomasney, J. W.; Stupp, S. I. Nano Lett. 2006, 6, 2086-2090.

(9) Guerrini, M.; Beccati, D.; Shriver, Z.; Naggi, A.; Viswanathan, K.; Bisio, A.; Capila, I.; Lansing, J. C.; Guglieri, S.; Fraser, B.; Al-Hakim, A.; Gunay, N. S.; Zhang, Z.; Robinson, L.; Buhse, L.; Nasr, M.; Woodcock, J.; Langer, R.; Venkataraman, G.; Linhardt, R. J.; Casu, B.; Torri, G.; Sasisekharan, R. Nat. Biotechnol. 2008, 26, 669-675.

(10) Mammadov, R.; Mammadov, B.; Toksoz, S.; Aydin, B.; Yagci, R.; Tekinay, A. B.; Guler, M. O. Biomacromolecules 2011, 12, 35083519 .

(11) Fairbrother, W. J.; Champe, M. A.; Christinger, H. W.; Keyt, B. A.; Starovasnik, M. A. Structure 1998, 6, 637-648.

(12) Stauffer, M. E.; Skelton, N. J.; Fairbrothe, W. J. J. Biomol. NMR 2002, 23, 57-61.

(13) Mizuno, K.; Inoue, H.; Hagiya, M.; Shimizu, S.; Nose, T.; Shimohigashi, Y.; Nakamura, T. J. Biol. Chem. 1994, 269, 1131-1136.
(14) Zhou, H.; Mazzulla, M. J.; Kaufman, J. D.; Stahl, S. J.; Wingfield, P. T.; Rubin, J. S.; Bottaro, D. P.; Byrd, R. A. Structure 1998, 6, 109116.

(15) Schlessinger, J.; Plotnikov, A. N.; Ibrahimi, O. A.; Eliseenkova, A. V.; Yeh, B. K.; Yayon, A.; Linhardt, R. J.; Mohammadi, M. Mol. Cell 2000, 6, 743-750.

(16) Thompson, L. D.; Pantoliano, M. W.; Springer, B. A. Biochemistry 1994, 33, 3831-3840.

(17) Ruppert, R.; Hoffmann, E.; Sebald, W. Eur. J. Biochem. 1996, 237, 295-302.

(18) Alberts, B. Molecular Biology of the Cell, 4th ed.; Garland Science: New York, 2002.

(19) Simons, M.; Rubanyi, G. M. Modern Concepts in Angiogenesis; Imperial College Press: London, 2007.

(20) Kwan, C. P.; Venkataraman, G.; Shriver, Z.; Raman, R.; Liu, D.; Qi, Y.; Varticovski, L.; Sasisekharan, R. J. Biol. Chem. 2001, 276, 23421-23429.

(21) Iozzo, R. V.; San Antonio, J. D. J. Clin. Invest. 2001, 108, 349355.

(22) Pellegrini, L. Curr. Opin. Struct. Biol. 2001, 11, 629-634.

(23) Zhou, Z.; Wang, J.; Cao, R.; Morita, H.; Soininen, R.; Chan, K. M.; Liu, B.; Cao, Y.; Tryggvason, K. Cancer Res. 2004, 64, 4699-4702.

(24) Figg, W. D.; Folkman, J. Angiogenesis: An Integrative Approach from Science to Medicine, 1st ed.; Springer: New York, 2008.

(25) Chu, H.; Johnson, N. R.; Mason, N. S.; Wang, Y. J. Controlled Release 2011, 150, 157-163.

(26) Spooner, B. S.; Holladay, C. R. Cell Motil. 1981, 1, 167-178.

(27) Kwon, S. E.; Chapman, E. R. Neuron 2011, 70, 847-854.

(28) Liebau, S.; Vaida, B.; Storch, A.; Boeckers, T. M. Stem Cells 2007, 25, 1720-1729.

(29) Mammadov, B.; Mammadov, R.; Guler, M. O.; Tekinay, A. B. Acta Biomater. 2012, 8, 2077-2086.

(30) Ashikari-Hada, S.; Habuchi, H.; Kariya, Y.; Itoh, N.; Reddi, A. H.; Kimata, K. J. Biol. Chem. 2004, 279, 12346-12354.

(31) Ibrahimi, O. A.; Zhang, F.; Hrstka, S. C.; Mohammadi, M.; Linhardt, R. J. Biochemistry 2004, 43, 4724-4730. 